Research Paper

\title{
Antigen presentation of the Oct4 and Sox 2 peptides by CD154-activated B lymphocytes enhances the killing effect of cytotoxic $T$ lymphocytes on tumor stem-like cells derived from cisplatin-resistant lung cancer cells
}

\author{
Xueyan Zhang*, Yanwei Zhang*, Jianlin Xu, Huimin Wang, Xiaoxuan Zheng, Yuqing Lou, Baohui Han ${ }^{\bowtie}$ \\ Department of Pulmonary, Shanghai Chest Hospital, Shanghai Jiaotong University, Shanghai 200030, China \\ * These authors contributed equally to this work. \\ $\square$ Corresponding author: Baohui Han, Department of Pulmonary, Shanghai Chest Hospital, Shanghai Jiaotong University, Shanghai 200030, China Fax: \\ +86-21-62821990 Email: xkyyhan@gmail.com \\ (c) Ivyspring International Publisher. This is an open access article distributed under the terms of the Creative Commons Attribution (CC BY-NC) license \\ (https://creativecommons.org/licenses/by-nc/4.0/). See http://ivyspring.com/terms for full terms and conditions.
}

Received: 2017.05.02; Accepted: 2017.11.21; Published: 2018.01.01

\begin{abstract}
The present study investigated whether antigen presentation of the Oct4 and Sox2 peptides by CD154-activated B lymphocytes can enhance the killing effect of CD8 $8^{+}$cytotoxic T lymphocytes (CTLs) on lung stem-like cancer cells (SLCCs). The CTLs were generated using an accelerated co-cultured dendritic cells $(\mathrm{DC})(\mathrm{acDC})$ assay by incubating human peripheral blood mononuclear cells (PBMCs) from non-small-cell lung cancer patients with antigen peptides of Oct4 and Sox2 in the presence of several DC-activating agents. CD154+ NIH3T3 cells prepared by CD154 lentiviral transfection were used as feeder layer to activate primary B cells $\left(\mathrm{CD} 19^{+}\right)$obtained from PBMCs. Activated B cells were co-cultured with CTLs to present antigen peptides of Oct4 and Sox2. CTLs co-cultured with activated B cells were evaluated for the levels of secreted inflammatory cytokines using ELISA. In addition, the killing effect of the CTLs on SLCCs derived from cisplatin-resistant strain of human lung cancer cell line PC9 was evaluated by flow cytometry using CFSE labeling of the target cells. After the acDC assay, the PBMCs exhibited a significant $(p<0.01)$ increase in the population of $C D 8^{+} / C D 3^{+}$cells, indicating successful preparation of CTLs. The primary $B$ cells cultured on the $\mathrm{CD} 154^{+} \mathrm{NIH} 3 \mathrm{~T} 3$ feeder layer resulted in significant $(p<0.01)$ increase in the proportions of population expressing CD80, CD86, or HLA-A, indicating successful activation of the B cells. The co-culture of CTLs with CD154-activated B cells presenting the Oct4 and Sox2 peptides caused significant increase in the levels of secretory inflammatory cytokines and exhibited enhanced killing of the SLCCs derived from cisplatin-resistant PC9 cells. Antigen presentation of the Oct 4 and Sox2 peptides by CD154-activated B cells can enhance the killing effect of CTLs towards lung SLCCs.
\end{abstract}

Key words: antigen presentation; cisplatin-resistant; cytotoxic T lymphocytes; lung stem-like cancer cell; Oct4; Sox2.

\section{Introduction}

The conventional methods for cancer treatment such as chemotherapy and surgery offer limited success in late stage cancer patients [1]. As an alternative approach, $\mathrm{CD} 8^{+}$cytotoxic $\mathrm{T}$ lymphocytes (CTLs) immunotherapy has evolved as a potential candidate for elimination of several tumor variants [2-5]. The effector CTLs mediate selective apoptosis of the target cells through perforin/granzyme and Fas/tumour necrosis factor mediated mechanisms [2], but the success of the tumor-directed CTLs greatly depend on their adequate number and unimpaired functionality [6]. Therefore, there is a constant need for investigation of immunomodulation that could produce tumor-directed CTLs with enhanced efficacy. 
Traditionally, the adaptive cellular immunotherapy approach transfuses in vitro-induced tumor specific CTLs into patients. This approach depends on a host of isolation of antigen-presenting cells (APCs) or dendritic cells (DCs) from peripheral blood of patients to timulate the maturation of naïve $\mathrm{T}$ lymphocytes $[7,8]$. Nevertheless, this method is limited by the amount of blood that can be collected from the cancer patients. In addition, the number of DCs in the peripheral blood is very limited and DCs are hard to amplify in vitro [9]. Therefore, the application of adoptive cell therapy in the clinical treatment of cancer patients is greatly limited. B cells in peripheral blood possess antigen presenting function and they are easy to culture and amplify in vitro. Therefore, B cells are gaining significance and popularity as the main APCs for activating CTLs instead of DCs [10, 11]. In addition, the activated B cells can be expanded exponentially for several weeks at high purity without loss of the antigen-presenting function, providing an almost unlimited source of cellular adjuvant [12].

The main reason for tumor multidrug resistance (MDR) and failure of chemotherapy and radiotherapy is the presence of surviving stem-like cancer cells (SLCCs) [13]. Lung cancer patients face high rate of recurrence even after definitive therapy, mainly due to the presence of SLCCs $[14,15]$. Immunothereapy has been used to treat lung cancer albeit with limited success [16]. CTL-mediated immunotherapy is a promising approach for further development of therapeutic approaches in lung cancer owing to its selective apoptotic effect on target cells [17, 18]. Identification of the markers on SLCCs is the prerequisite for successful CTL therapy. Several markers for different cancer variants have been studied, including ALDH1A1, ALDH, CD44, CD133, and HER2 [8]. In lung SLCCs, Oct4 and Sox2 are closely related to tumor MDR [19]. Overexpression of Oct4 can promote the differentiation of lung cancer cells into SLCCs and acquisition of drug resistance, whereas transduction of Oct4 shRNA could restore the sensitivity of cancer cells to chemotherapy [20]. Therefore, CTLs targeting Oct 4 and Sox 2 is a potential approach to kill MDR SLCCs, to reduce recurrence and metastasis, and thereby to improve the prognosis.

Therefore, we carried out the present study to investigate if antigen presentation of the Oct4 and Sox2 peptides by CD154-activated B lymphocytes could enhance the immunocompetence of CTLs.

\section{Materials and Methods}

\section{Experimental design}

Human peripheral blood mononuclear cells
(PBMCs) were isolated by Ficoll-Hypaque (Amersham, Freiburg, Germany) density gradient centrifugation from heparinized blood samples of 24 patients with newly diagnosed stage IIIB-IV non-small-cell lung cancer (treatment-naïve) at the Department of Respiratory Medicine, Shanghai Chest Hospital $(8-10 \mathrm{ml} /$ patient, 24 patients, including 13 males and 11 females; mean age of 58 years). As the amount of cells required for the experiment is large, the amount of cells from one patient is not sufficient to carry out the experiment. Therefore, the cells separated from the blood samples of multiple patients were pooled before the subsequent experiments. The study was approved by the ethics committee of Shanghai Chest Hospital, and informed written consents were obtained from the patients.

The PBMCs were subjected to accelerated co-cultured DC (acDC) assay by incubating with antigen peptides of Oct4 and Sox 2 in the presence of several DC-activating agents, as previously described [21]: TNF- $\alpha(1000 \mathrm{U} / \mathrm{mL}), \mathrm{IL}-1 \beta(10 \mathrm{ng} / \mathrm{mL})$, and IFN- $\gamma \quad(1000 \mathrm{U} / \mathrm{mL}$, all from R\&D Systems); prostaglandin $\mathrm{E}_{2}\left(\mathrm{PGE}_{2} ; 1 \mu \mathrm{M}\right.$; Merck Calbiochem); CpG ODN2216 (5 $\mu \mathrm{g} / \mathrm{mL}$; Cell Sciences $)$, polyinosinic:polycytidylic acid (polyI:C, $20 \mu \mathrm{g} / \mathrm{mL}$; Cayla/InvivoGen); lipopolysaccharide (LPS, 100 $\mathrm{ng} / \mathrm{mL}$; from $E$ coli 055:B5; Sigma-Aldrich); and anti-CD40 mAb (1 $\mu \mathrm{g} / \mathrm{mL}$; clone G28.5) and IFN- $\mathrm{a}-2 \mathrm{a}$ (1000 U/mL; Roferon-A; Roche). The procedure induces DC maturation and promotes the sequential steps of $\mathrm{T}$ cell differentiation [21]. Following the acDC assay, the expression of $\mathrm{T}$ cell surface markers was detected and the $\mathrm{CD}^{+} / \mathrm{CD}^{+} \mathrm{T}$ cells (CTLs) were isolated using magnetic beads.

Viral vectors for the overexpression of CD154 packaged in HEK293T cells were used to transfect NIH3T3 cells. The CD154 ${ }^{+}$NIH3T3 cells were used as the feeder layer to activate primary B cells $\left(\mathrm{CD} 19^{+}\right)$ obtained from PBMCs. The CD154-activated B cells were used to present antigen peptides of Oct4 and Sox2 to CTLs. CTLs not receiving antigen presentation with CD154-activated B cells were used as control. The control and activated CTLs were compared with respect to the secretion levels of inflammatory cytokines and killing effect on SLCCs derived from a cisplatin-resistant strain of the human lung cancer cell line PC9.

\section{Preparation of cisplatin-resistant PC9-derived SLCCs}

The human lung cancer cell line PC9 was obtained from the Cell Bank of the Chinese Academy of Sciences, Shanghai. The cells were cultured in Dulbecco's modified Eagles medium (DMEM) supplemented with 10\% fetal bovine serum (FBS), 100 
$\mathrm{U} / \mathrm{mL}$ penicillin, and $100 \mu \mathrm{g} / \mathrm{mL}$ streptomycin in a $37^{\circ} \mathrm{C}$ humidified incubator with $5 \% \mathrm{CO}_{2}$.

The cisplatin-resistant PC9 cell line was established by gradient induction using increasing concentrations of cisplatin. Briefly, PC9 cells at $80 \%$ confluence were exposed to $1 \mu \mathrm{mol} / \mathrm{L}$ cisplatin and cultured at $37^{\circ} \mathrm{C}$ with $5 \% \mathrm{CO}_{2}$ for $24 \mathrm{~h}$. The culture media was replaced by drug-free fresh culture medium until the cells reached a confluence of $70-80 \%$. The procedure was repeated with increased concentration of cisplatin at $2.5 \mu \mathrm{mol} / \mathrm{L}$ followed by 5 $\mu \mathrm{mol} / \mathrm{L}$. At the end of the induction, the cells were cultured for 1-2 months with $10 \mu \mathrm{mol} / \mathrm{L}$ of cisplatin to obtain the PC9 cisplatin-resistant cell line.

This pool of cisplatin-resistant PC9 cells was used to derive SLCCs. The cisplatin-resistant PC9 cells in the logarithmic growth phase were inoculated into 6 -well plates at a density of $1 \times 10^{3} / \mathrm{mL}$. The suspension culture was performed with serum-free stem cells culture medium (DMEM-F12 medium; insulin, 4U/L; B27, 1×; EGF, $20 \mathrm{ng} / \mathrm{mL}$; bFGF, 20 $\mathrm{ng} / \mathrm{mL}$ ), which induced the formation of stem-like cell spheres. Tumor cells were observed to form stem-like cell spheres after 2-3 weeks. The cells were passaged to the third generation of stem-like cell spheres for subsequent experiments.

\section{Induction of $T$ cell differentiation with acDC assay}

The PBMCs $\left(5 \times 10^{5} /\right.$ well, 96-well plate) were incubated with antigen peptides of Oct4 and Sox2 (both $5 \mu \mathrm{g} / \mathrm{ml}$ ) in the presence of GM-CSF $(500 \mathrm{U} / \mathrm{ml})$ and interleukin (IL)-4 (10 ng/ml) for $24 \mathrm{~h}$. The volume was $200 \mu \mathrm{l}$. Then, TNF-a $(2 \mathrm{ng} / \mathrm{ml}$, final concentration), IL-1 (2 ng/ml, final concentration), and PGE $_{2}(0.2 \mu \mathrm{M}$, final concentration) were added into the system $(250 \mu \mathrm{l})$ for incubation for $24 \mathrm{~h}$. Next, two fifths of the culture medium was replaced with fresh medium supplemented with IL-2 $(40 \mathrm{U} / \mathrm{ml}$, final concentration $)$ and IL-7 $(20 \mathrm{ng} / \mathrm{ml}$, final concentration). The cells were cultured for 5 days. The Oct4 antigen polypeptide sequence was DVVRVWFCNRRQKGK. The Sox2 antigen polypeptide sequence was PWRTMDASERGRLLYKLADLIERD.

\section{Transfection of NIH3T3 cells with lentivirus carrying CD154}

The HEK293T cells were obtained from the American Type Culture Collection (Rockville, MD). The NIH3T3 cells were obtained from the Cell Bank of the Chinese Academy of Sciences (Shanghai). The cells were cultured in Dulbecco's modified Eagles medium (DMEM) supplemented with 10\% FBS and $100 \mathrm{U} / \mathrm{mL}$ penicillin and $100 \mu \mathrm{g} / \mathrm{mL}$ streptomycin in a $37^{\circ} \mathrm{C}$ humidified incubator with $5 \% \mathrm{CO}_{2}$.

CD154 fragment was constructed by PCR with specific primers designed from the mouse genome library and sub-cloned into the lentiviral vector LV5. The lentiviral particles were produced by performing transient co-transfection involving a four-plasmid expression system (LV5, PG-p1-VSVG, PG-P2-REV, and PG-P3-RRE) in HEK293T cells according to the manufacturer's instructions, and concentrated through ultracentrifugation, as described previously [5].

The NIH3T3 cells were seeded into 24-well plates at a density of $5 \times 10^{4}$ / well in $500 \mu \mathrm{l}$ of complete DMEM medium. After incubation for $24 \mathrm{~h}$, the cells were treated with Lenti/CD154 (103 vp per cell) for 24 $\mathrm{h}$ at $37^{\circ} \mathrm{C}$ and $5 \% \mathrm{CO}_{2}$. Then, the NIH3T3 cells were subsequently maintained in fresh complete DMEM medium. As a control, NIH3T3 cells with Lenti/GFP were used.

\section{Activation of primary B cells with CD 154+ NIH3T3 feeder layer}

The $\mathrm{CD} 19^{+} \mathrm{B}$ cells were isolated from PBMCs using Dynabeads Untouched Human B Cells (Life Technologies, NY, USA), according to the manufacturer's instructions. The isolated $\mathrm{CD}_{19}{ }^{+} \mathrm{B}$ cells were cultured in the IMDM culture medium supplemented with 10\% FCS, $2 \%$ ITS, 100 U/ml IL-2, $40 \mathrm{ng} / \mathrm{ml} \mathrm{IL}-4$, and $1 \mu \mathrm{g} / \mathrm{ml}$ cyclosporine A.

The CD154+ NIH3T3 cells were used as a feeder layer to culture and activate the B cells. The CD154+ NIH3T3 cells were seeded in $40-\mathrm{mm}$ culture plate $\left(5 \times 10^{4}\right.$ cells $\left./ \mathrm{cm}^{2}\right)$ after radiation treatment with $30 \mathrm{~Gy}$. After incubation for $24 \mathrm{~h}, 1 \mathrm{~mL}$ of B cells $\left(2 \times 10^{5} / \mathrm{cm}^{2}\right)$ with $1 \mathrm{~mL}$ of $2 \times \mathrm{B}$ cell culture medium were inoculated on the adherent feeder layer cells. After 3-5 days, the suspended B cells were collected, and newly prepared feeder layer was replaced to continue culture. These pools of cells were used as activated B cells.

\section{Antigen presentation of the Oct4 and Sox2 peptides by CD154-activated B cells to CTLs}

The CD154-activated B cells $\left(1 \times 10^{6}\right)$ were loaded with $10 \mu \mathrm{g}$ of the antigen polypeptide of Oct4 and Sox2 as experiment group and CMV pp65 peptide as positive control group, and were subsequently incubated for $2 \mathrm{~h}$. Then, the cells received radiation treatment (30 Gy), and were washed and resuspended with IMDM medium containing $10 \%$ FCS. Next, they were co-cultured with CTLs obtained from the acDC assay at a ratio of $1: 1$ for 3 days. After that, the medium was renewed with supplementation of IL-2 (50 U/ml, final concentration) and IL-7 $(25 \mathrm{ng} / \mathrm{ml}$, final concentration). The culture lasted for 4 days. The 
above steps were repeated once; then the CTLs were collected and again subjected to antigen presentation by CD154-activated $B$ cells at a ratio of 10:1 for 1 week, in the presence of CD3 antibody $(1 \mu \mathrm{g} / \mathrm{ml})$ and IL-2 (500 U/ml).

\section{Evaluation of the CTL killing activity}

The target cells (cisplatin-resistant PC9-derived SLCCs) were labeled with carboxyfluorescein succinimidyl ester (CFSE) (AAT Bioquest, USA) and seeded into 6 well plates $\left(1 \times 10^{5}\right.$ cells/well). CTLs receiving Oct4 and Sox2 antigen presentation with CD154-activated B cells were seeded into the wells at different ratios (CTLs: SLCCs derived from cisplatin-resistant PC9 cells $=$ 20:1, 10:1, and 5:1). CTLs receiving CMV pp65 antigen presentation were regarded as positive control (CTLs: SLCCs $=20: 1$ ), and CTLs without antigen presentation were taken as blank control (CTLs: SLCCs =20: 1). The cells were co-cultured at $37^{\circ} \mathrm{C}$ for $4 \mathrm{~h}$. Then, $10 \mu \mathrm{l}$ of $\mathrm{PE}$ fluorescent dye (BD, USA) was added into each well. $\mathrm{CFSE}^{+} \mathrm{PE}^{+}$cells were considered as the killed target cells. The proportions of CFSE ${ }^{+}$cells and $\mathrm{CFSE}^{+} \mathrm{PE}^{+}$ cells were estimated by flow cytometry analysis. The percentage of cytotoxic activity was calculated using the formula, $\mathrm{CFSE}^{+} \mathrm{PE}^{+}$cell number $/ \mathrm{CFSE}^{+}$cell number $\times 100 \%$.

\section{Flow cytometry analysis}

Besides the proportions of $\mathrm{CFSE}^{+}$cells and $\mathrm{CFSE}^{+}$ $\mathrm{PE}^{+}$cells (see above), the cell surface markers with fluorescent labeling in other assays were also detected using FACS Vantage SE flow cytometry (BD, USA). On the $6^{\text {th }}$ day after CD154-LV5 transfection, the proportion of CD154+ NIH3T3 cells was measured by staining with CD154-PE antibody (Ebiosence, USA). Primary B cells, which were individually cultured for 3 weeks or after 3 weeks of culture on the feeder layer, were stained with HLA-A-PE, CD80-PE, or CD86-APC antibodies (Ebiosence, USA). PBMCs undergoing acDC assay and the control were stained with CD3-APC or CD8-PE antibodies (Ebiosence, USA).

\section{Enzyme-linked immunosorbent assay (ELISA)}

The supernatants were obtained from the culture of blank control CTLs alone, CMV pp65 control CTLs (receiving the antigen presentation with CD154-activated B cells by CMV pp65), and Oct4 and Sox2 CTLs (receiving the antigen presentation with CD154-activated B cells by Oct4 and Sox2). The supernatant was analyzed for human interferon gamma (IFNY) and IL-2 secretion levels with commercially available ELISA kits (MultiSciences, China), according to the manufacturer's instruction. The reaction was read using a microplate reader
(Bio-Tek Instruments, Winooski, VT, USA) at $450 \mathrm{~nm}$.

\section{Immunofluorescence staining}

Immunofluorescence staining for Oct 4 and Sox2 was performed on cell slides of cisplatin-resistant PC9 cells and cisplatin-resistant PC9-derived SLCCs. The cell slides were fixed with $4 \%$ paraformaldehyde, permeabilized with $0.5 \%$ Triton X-100, and blocked with $6 \%$ normal goat serum. The slides were incubated overnight at $4^{\circ} \mathrm{C}$ with 1:20 rabbit anti-human Oct4 or Sox2 antibody (Abcam, USA). After washing with PBS the slides were incubated either with 1:800 Alexa Fluor 647 (for Sox2) or FITC (for Oct4) labeled goat anti-rabbit antibody at $37^{\circ} \mathrm{C}$ for 30 min followed by treatment with DAPI. The slides were washed with PBS and blocked with anti-fluorescent quencher. The images were collected using a laser scanning focusing microscope (Bio-Tek, USA).

\section{Statistical analysis}

Statistical analysis was performed using SPSS 19.0. Continuous data were expressed as the mean \pm standard deviation (S.D) and compared using one way ANOVA followed by the HSD post hoc test. A P-value $<0.05$ was considered to be statistically significant.

\section{Results}

The average percentage of $\mathrm{CD} 154^{+}$cells among Lenti/CD154-transfected NIH3T3 cells was $>99 \%$, while that of Lenti/GFP-transfected cells was only around 15\% (Supplementary Figure 1). After culture on feeder layer of $\mathrm{CD}_{154^{+}}{ }^{\mathrm{NIH}} 3 \mathrm{T3}$ cells, the populations of $\mathrm{B}$ cells expressing CD80, CD86, or HLA-A were all significantly increased (Supplementary Figure 2). These results demonstrated that the primarily cultured B cells were successfully activated by using CD $154^{+}$NIH3T3 cells as the feeder layer.

The cisplatin-resistant PC9 cells were cultured in the presence of serum-free stem cells culture medium that led to the formation of spheroid aggregates (Supplementary Figure 3A). The cells passaged to the third generation of stem-like cell spheres were considered as SLCCs and used for further investigation. The SLCCs obtained from the stem-like cell sphere were characterized by immunofluorescence staining of Oct4 and Sox2 (Supplementary Figure 3B).

After the acDC assay, the percentages of $\mathrm{CD}^{+}$ and $\mathrm{CD}^{+}$populations in PBMCs were markedly increased (Figure 1). After antigen presentation of the Oct 4 and Sox 2 peptides by CD154-activated B cells, the secretion of IL-2 and IFNY were significantly 
increased in Oct4 and Sox2 CTLs group compared with CMV pp65 positive control group and blank control group (Figure 2A). The killing effect of Oct4 and Sox2 specific CTLs on SLCCs derived from cisplatin-resistant PC9 cells increased significantly either in CTLs:SLCCs at 20:1, 10:1, or 5:1 compared with CMV pp65 positive control and blank control (Figure 2B).

\section{Discussion}

The efficacy of targeted CTLs on killing of SLCCs depends greatly on the APCs. In the present study, we demonstrate that CD154-activated B cells could effectively present peptides of Oct 4 and Sox 2 to CTLs, leading to a significant increase in the levels of secreted inflammatory cytokines by CTLs and enhanced CTL-mediated killing of cisplatin-resistant lung cancer cells.

In lung cancer, MDR leads to recurrence and metastasis, and thereby poor prognosis of patients with lung cancer [22]. The key to control the progression of the disease and improve the prognosis of the patients lies in effectively killing the drug-resistant cancer cells. Since the SLCCs are resistant to cytotoxic drugs and targeted therapies such as EGFR tyrosine kinase inhibitors, specific CTL-mediated immunotherapy could be an effective choice for the treatment of drug-resistant SLCCs.

Efficient antigen presentation is a prerequisite for the development of a T-cell-mediated immune response in vitro and in vivo. CD154, also called CD40 ligand (CD40L), is primarily expressed on activated $\mathrm{T}$ cells [23]. It binds to CD40 on APCs, which leads to many effects depending on the target cell type [24]. CD154 promotes B cell maturation and function by engaging $\mathrm{CD} 40$ on the $\mathrm{B}$ cell surface and therefore facilitating cell-cell communication [25]. CD40 and CD154 are both members of the TNF family, and their interaction provides a potent signal for the activation of B lymphocytes [26]. Therefore, B cells activated by CD154/CD40 are a promising alternative to DCs as APCs for immunotherapy [12].
A

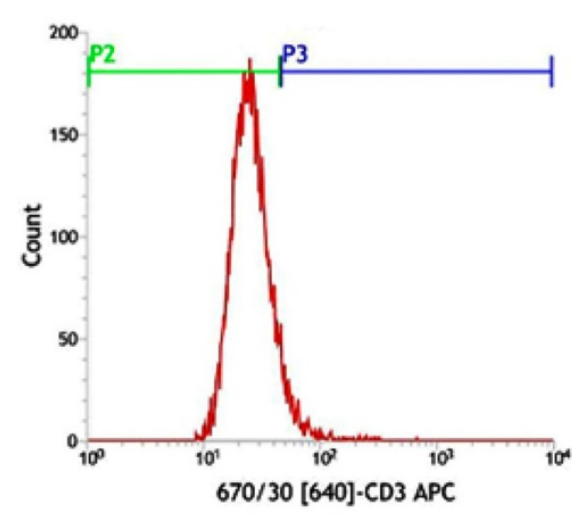

B

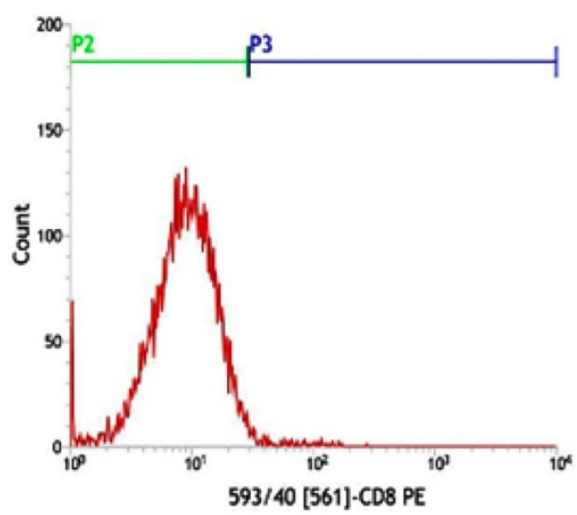

PBMCs after acDC assay
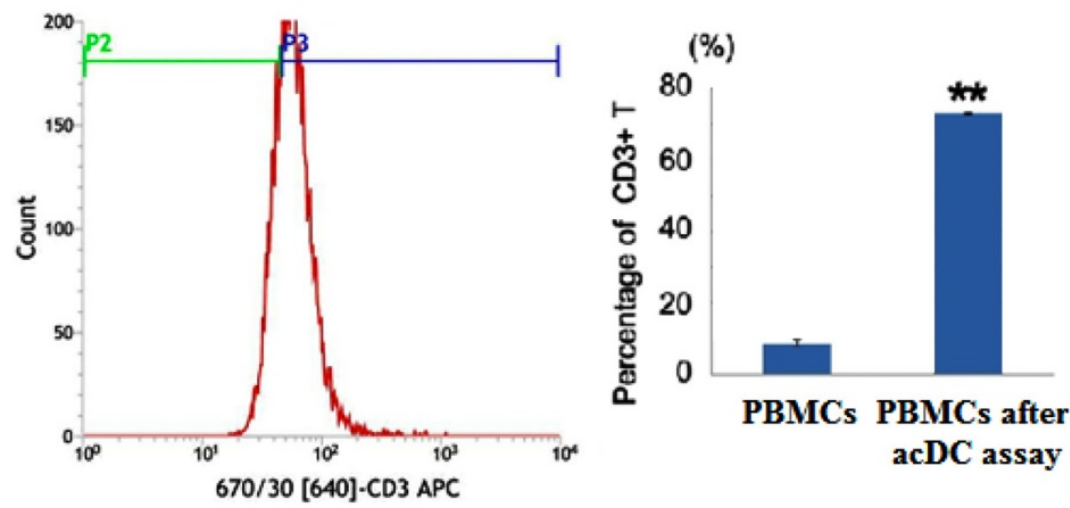

Figure 1. The "accelerated co-cultured dendritic cell (acDC)" assay promoted peripheral blood mononuclear cells (PBMCs) to differentiate into cytotoxic T lymphocytes (CTLs) Human PBMCs from non-small-cell lung cancer patients were incubated with antigen peptides of Oct4 and Sox2 in the presence of several DC-activating agents, which was called the acDC assay. At the end of this assay, the $T$ cell surface markers were detected by flow cytometry analysis. PBMCs not undergoing acDC assay were used as control. And PBMCs after acDC assay group presented with both increased percentages of CD3 ${ }^{+}$cell (A) and CD8+ cells (B) compared with PBMCs without acDC assay group. ${ }^{* *} p<0.01$, compared with PBMCs (without acDC assay) 
A
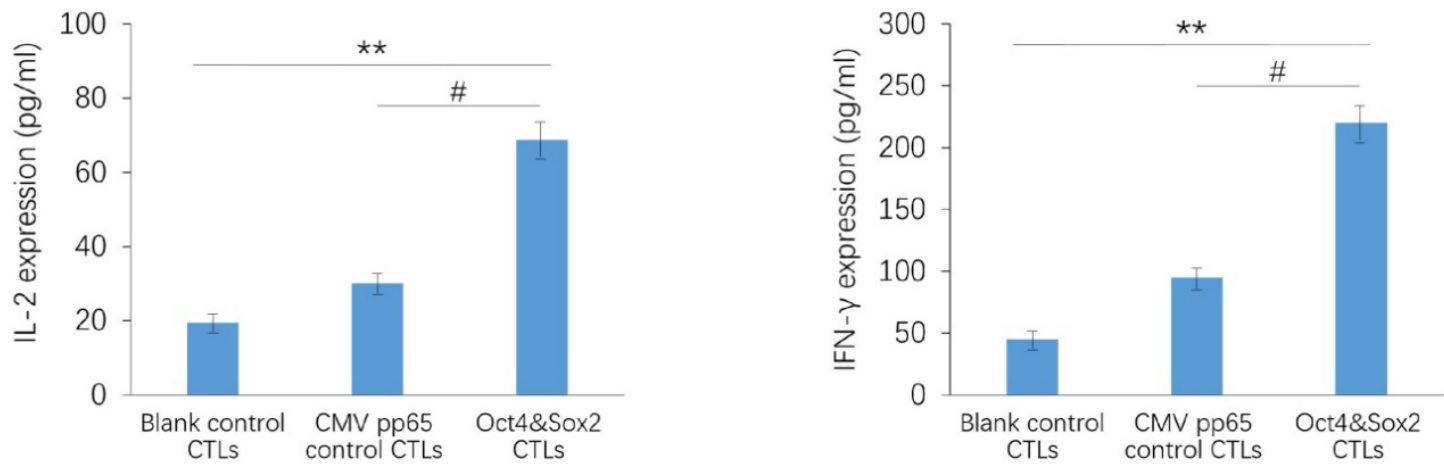

B

Oct4 and Sox2 specific CTLs + SLCCs

$20: 1$

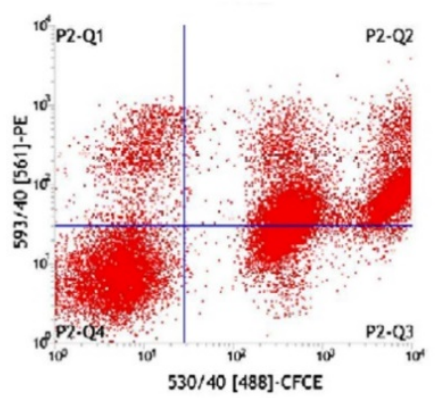

Blank control CTLs + SLCCs $20: 1$

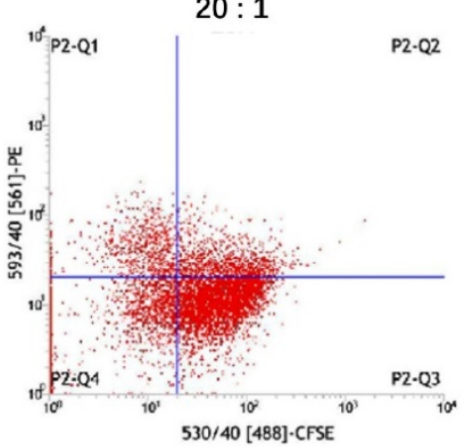

$10: 1$

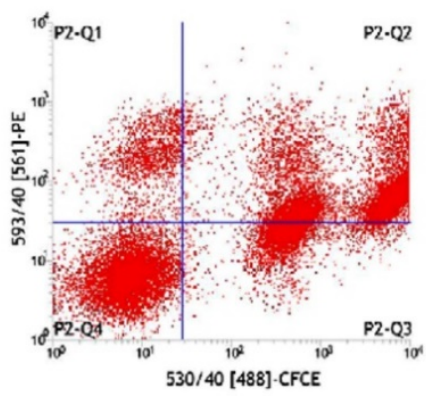

CMV pp65 control CTLs + SLCCs

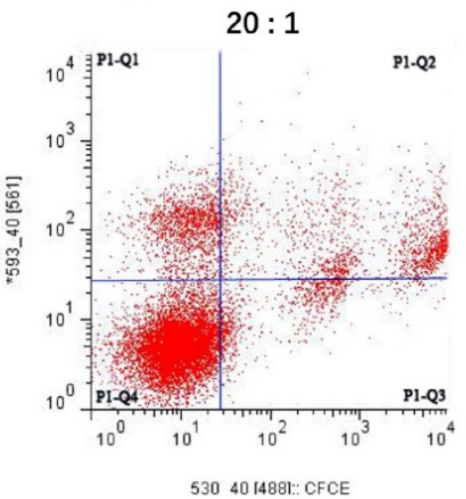

$5: 1$
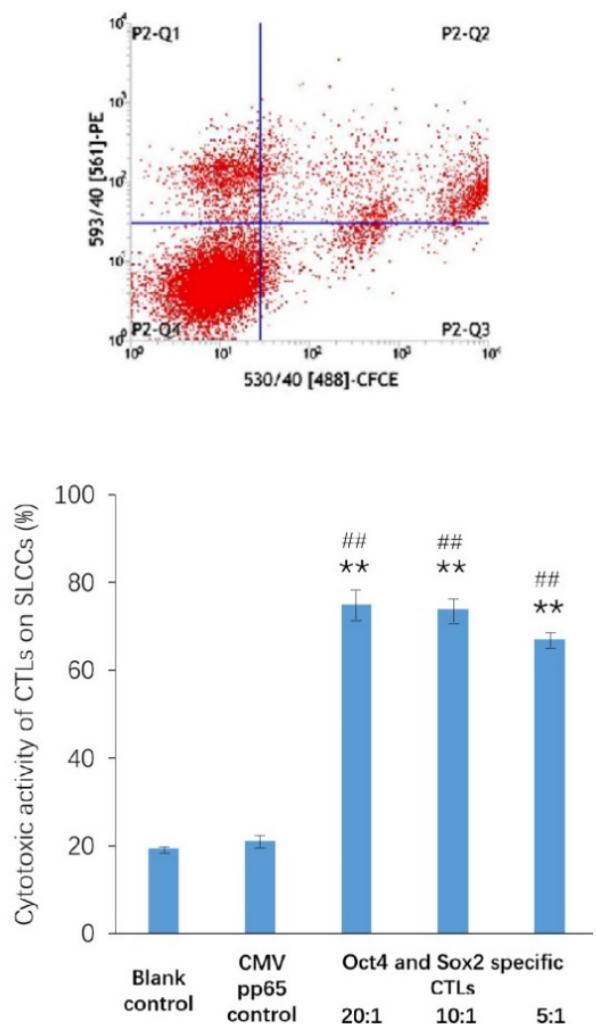

Figure 2. Antigen presentation of the Oct4 and Sox 2 peptides by CD154-activated B cells enhances the immunocompetence of cytotoxic T lymphocytes (CTLs). CTLs were isolated from the accelerated co-cultured dendritic cell (acDC) assay. CD154-activated B cells, Oct4 and Sox2 polypeptide were co-cultured with CTLs (Oct4 and Sox2 specific CTLs group), while CTLs co-cultured with CD154-activated B cells and CMV pp65 peptide were set as positive control, and CTLs cultured alone were taken as blank control. These three kinds of CTLs were compared with regard to the secretion levels of inflammatory cytokines, as measured by ELISA, which disclosed that IL-2 and INF- $\gamma$ were both increased in Oct4 and Sox2 CTLs group compared with blank control and CMV pp65 control groups (A). Their killing effects on stem-like cancer cells (SLCCs) derived from a cisplatin-resistant strain of the human lung cancer cell line PC9 were also compared, which illuminated that Oct4 and Sox2 specific CTLs achieved a higher killing effect (CTLs: SLCCs= 20:1, 10:1 and 5:1) compared to both CMV pp65 control (CTLs: SLCCs= 20:1) and blank control (CTLs: SLCCs= 20:1) (B). CTLs were co-cultured with target cells labeled with carboxyfluorescein succinimidyl ester (CFSE) at a ratio of for $4 \mathrm{~h}$. Then, PE fluorescent dye was added. CFSE ${ }^{+} \mathrm{PE}^{+}$cells were considered as the killed target cells. The proportions of CFSE+ cells and CFSE+ PE+ cells were estimated by flow cytometry. The percentage of cytotoxic activity was calculated as CFSE ${ }^{+} \mathrm{PE}^{+}$cell number /CFSE ${ }^{+}$cell number $\times 100 \%$. ** $p<0.01$, compared with blank control. \#<0.05, \#<0.01, compared with CMV pp65 positive control.

Although CD40 activation dramatically improves antigen presentation by normal and malignant $B$ cells, efficiently inducing naïve and memory $\mathrm{CD}^{+}$and $\mathrm{CD}^{+}{ }^{+} \mathrm{T}$-cell responses through CD40 activation presents practical difficulties. The generation of activated $\mathrm{CD}^{+} \mathrm{T}$ cells as the feeder layer cells for CD40 B cells is complicated not only for cell therapy but also for routine laboratory research [27]. CD154 is transiently expressed on the surface of activated $\mathrm{CD}^{+} \mathrm{T}$ cells for less than $24 \mathrm{~h}$. Therefore, a 
simplified method for the generation of $\mathrm{CD} 154^{+}$cells is urgently needed. In the present study, we transduced CD154 into NIH3T3 cells with lentivectors to generate $\mathrm{CD} 154^{+} \mathrm{NIH} 3 \mathrm{T3}$ cells. In the parental NIH3T3 cells, the expression of CD154 is very low, only $14.8 \%$. After lentivectors-mediated CD154 transduction, the rate of $\mathrm{CD} 154^{+}$cells increased to $99.7 \%$, indicating that the lentivector is a good delivering tool for CD154, and that the CD154+ NIH3T3 cells could be used as the feeder layer cells for the induction of CD154-activated B cells in vitro.

In the present study, we demonstrated that when CD154-activated B cells are used to present the peptides of Oct4 and Sox2, it leads to dramatic increases in the secreted IFNY and IL2 levels by CTLs. These results indicate that the in vitro generation and expansion of Oct4/Sox2-specific CTLs using CD154-activated B cells is a feasible method for the treatment of lung cancer. The cytotoxicity of the CTLs presented with Oct 4 and Sox 2 by CD154-activated B cells was tested on MDR PC9-derived SLCCs. The CTLs exhibited enhanced cytotoxicity on the MDR PC9-derived SLCCs. The results are suggestive of CD154-activated B cells as an APC for CTLs as a probable therapeutic strategy for lung cancer. Nevertheless, these results will have to be confirmed in vivo as well as in vitro in additional target cancer cell lines.

Besides, cytotoxic activity of Oct4 and Sox2 specific CTLs on PC9 cells was also detected (Supplementary Figure 4). And we found after co-culturing as the ratio $20: 1,10: 1$ and 5:1, the cytotoxic activities were all above $80 \%$, which were comparatively higher than that of SLCCs to specific CTLs. The possible explanation might be that original lung cancer cells are more sensitive to various treatments than SLCCs including CTLs, thus CTLs would present with higher killing effect on PC9 cells than PC9 SLCCs. Although selective ability of CTLs for SLCCs is enhanced by Oct4/Sox2 antigens presenting procedure, the sensitivity of SLCCs to Oct4/Sox2 specific CTLs is still relative less than that of PC9 cells to CTLs.

In conclusion, the study demonstrates a simplified method to produce CD154+ NIH3T3 feeder layer cells for the activation of B cells in vitro. More importantly, the study suggests that the generation and expansion of Oct4/Sox2-specific CTLs by antigen presentation from CD154-activated B cells might be a potential method for the treatment of drug-resistant lung cancer.

\section{Supplementary Material}

Supplementary figures and tables.

http://www.jcancer.org/v09p0367s1.pdf

\section{Acknowledgements}

The study was supported by the National Natural Science Foundation of China (No.: 81502450).

\section{Compliance with Ethical Standards}

\section{Ethical approval}

All procedures performed in studies involving human participants were in accordance with the ethical standards of the institutional and/or national research committee and with the 1964 Helsinki declaration and its later amendments or comparable ethical standards.

\section{Informed consent}

Informed consent was obtained from all individual participants included in the study.

\section{Competing Interests}

The authors have declared that no competing interest exists.

\section{References}

1. Bonavida B, Chouaib S. Resistance to anticancer immunity in cancer patients: potential strategies to reverse resistance. Ann Oncol. 2017; 28: 457-467.

2. Maher J, Davies ET. Targeting cytotoxic $T$ lymphocytes for cancer immunotherapy. Br J Cancer. 2004; 91: 817-821.

3. Fousek K, Ahmed N. The Evolution of T-cell Therapies for Solid Malignancies. Clin Cancer Res. 2015; 21: 3384-3392.

4. June $\mathrm{CH}$. Adoptive $\mathrm{T}$ cell therapy for cancer in the clinic. J Clin Invest. 2007; 117: 1466-1476.

5. Wang Z, Li P, Xu Q, et al. Potent Antitumor Activity Generated by a Novel Tumor Specific Cytotoxic T Cell. PLoS One. 2013; 8: e66659.

6. Aerts JG, Hegmans JP. Tumor-specific cytotoxic T cells are crucial for efficacy of immunomodulatory antibodies in patients with lung cancer. Cancer Res. 2013; 73: 2381-2388.

7. Kwiatkowska-Borowczyk EP, Gabka-Buszek A, Jankowski J, et al. Immunotargeting of cancer stem cells. Contemp Oncol (Pozn). 2015; 19: A52-59.

8. Pan Q, Li Q, Liu S, et al. Concise Review: Targeting Cancer Stem Cells Using Immunologic Approaches. Stem Cells. 2015; 33: 2085-2092.

9. Kalinski P, Urban J, Narang R, et al. Dendritic cell-based therapeutic cancer vaccines: what we have and what we need. Future Oncol. 2009; 5:379-390.

10. Bennett SR, Carbone FR, Toy T, et al. B cells directly tolerize CD8(+) T cells. J Exp Med. 1998; 188: 1977-1983.

11. Mitchison NA. T-cell-B-cell cooperation. Nat Rev Immunol. 2004; 4: 308-312.

12. Wennhold K, Shimabukuro-Vornhagen A, Theurich S, et al. CD40-activated B cells as antigen-presenting cells: the final sprint toward clinical application. Expert Rev Vaccines. 2013; 12: 631-637.

13. Pattabiraman DR, Weinberg RA. Tackling the cancer stem cells - what challenges do they pose? Nat Rev Drug Discov. 2014; 13: 497-512.

14. Alamgeer M, Peacock CD, Matsui $W$, et al. Cancer stem cells in lung cancer: Evidence and controversies. Respirology. 2013; 18: 757-764.

15. Codony-Servat J, Verlicchi A, Rosell R. Cancer stem cells in small cell lung cancer. Transl Lung Cancer Res. 2016; 5: 16-25.

16. Holt GE, Podack ER, Raez LE. Immunotherapy as a strategy for the treatment of non-small-cell lung cancer. Therapy. 2011; 8: 43-54.

17. Mine $\mathrm{T}$, Gouhara $\mathrm{R}$, Hida $\mathrm{N}$, et al. Immunological evaluation of CTL precursor-oriented vaccines for advanced lung cancer patients. Cancer Sci. 2003; 94: 548-556.

18. Jadus MR, Natividad J, Mai A, et al. Lung cancer: a classic example of tumor escape and progression while providing opportunities for immunological intervention. Clin Dev Immunol. 2012; 2012: 160724.

19. Dogan I, Kawabata S, Bergbower E, et al. SOX2 expression is an early event in a murine model of EGFR mutant lung cancer and promotes proliferation of a subset of EGFR mutant lung adenocarcinoma cell lines. Lung Cancer. 2014; 85: 1-6.

20. Chiou SH, Wang ML, Chou YT, et al. Coexpression of Oct4 and Nanog enhances malignancy in lung adenocarcinoma by inducing cancer stem cell-like properties and epithelial-mesenchymal transdifferentiation. Cancer Res. 2010; 70: 10433-10444. 
21. Martinuzzi E, Afonso G, Gagnerault MC, et al. acDCs enhance human antigen-specific T-cell responses. Blood. 2011; 118: 2128-2137.

22. Holohan C, Van Schaeybroeck S, Longley DB, et al. Cancer drug resistance: an evolving paradigm. Nat Rev Cancer. 2013; 13: 714-726.

23. Durlanik S, Loyal L, Stark R, et al. CD40L expression by CD4+ but not CD8+ T cells regulates antiviral immune responses in acute LCMV infection in mice. Eur J Immunol. 2016; 46: 2566-2573.

24. Qin L, Qiu H, Zhang M, et al. Soluble CD40 ligands sensitize the epithelial ovarian cancer cells to cisplatin treatment. Biomed Pharmacother. 2016; 79: $166-175$

25. Brunekreeft KL, Strohm C, Gooden MJ, et al. Targeted delivery of CD40L promotes restricted activation of antigen-presenting cells and induction of cancer cell death. Mol Cancer. 2014; 13: 85.

26. $\mathrm{Xu}$ Y, Song $\mathrm{G}$. The role of CD40-CD154 interaction in cell immunoregulation. J Biomed Sci. 2004; 11: 426-438.

27. Ciantar JP, Mannering SI. An improved method for growing and analysing human antigen-specific CD4+ T-cell clones. Diabetes Metab Res Rev. 2011; 27: 906-912. 